\title{
Experimental study of liquid droplets impact on powder surface: The application of effective dimensionless parameters in analysis
}

\author{
Siqi $\mathrm{Li}^{*}$, Hourong $\mathrm{Yu}$, and Haisheng Fang \\ Huazhong University of Science and Technology, School of Energy and Power Engineering, 1037 \\ Luoyu Road, Wuhan, China
}

\begin{abstract}
Spreading dynamics of liquid droplets impacting onto powder bed are experimentally studied using high-speed photography. Dimensionless numbers- $W e, R e$, the modified $W e^{*}$ and $R e^{*}$ corrected by substrate deformation - are used to analyze the impact behaviors of droplets. The spreading time and the maximum spreading factor are further analyzed. The spreading time is accurately described by a universal scaling law that is obtained from the modified dimensionless time vs. the effective Weber number $\left(W e^{*}\right)$, and the maximum spreading factor is found to follow the modified classic scaling law $\beta_{\max }=f\left(W e^{*}, R e^{*}\right)$.

Keywords: Maximum spreading, Effective Weber number, Spreading time, Droplet impact.
\end{abstract}

\section{Introduction}

Droplet impact on powder beds occurs extensively in industrial and technological applications, such as granulation in the pharmaceutical industry ${ }^{[1,2]}$; granule formation by spraying for powder coating and spray drying ${ }^{[3,4]}$; and in three-dimensional printing processes $^{[5-7]}$. It is important to understand the dynamics of the droplet impact process on powder beds, which is critical for controlling such processes. Various impact behaviorse.g., spreading, retraction, rebounding, and splashing - and the resulting crater morphology as well as, liquid marbles have been investigated in detail ${ }^{[8-10]}$. The behaviors of droplets upon impact have been studied comprehensively by experimenta ${ }^{[11,12]}$, theoretical ${ }^{[13,14]}$, and numerical methods ${ }^{[15]}$.

In the experimental and theoretical study, the impact dynamics of liquid droplets and powder surfaces have been systematically investigated. The influences of initial impact velocity and liquid properties on spreading time and maximum spreading have been examined. By using modified Weber number and Reynolds number, we apply empirical expressions for non-deformable solid substrate to powder bed, and reveal their similarities for maximum spreading and spreading time. Scaling analyses were adopted to reveal three characteristic parameters.

\footnotetext{
* Corresponding author: m201971069@hust.edu.cn
} 


\section{Experimental setup and mathematical models}

Alumina beads were supplied by the manufacturer Hebei Xinda Alloy material, China. The initial packing fraction, $\Phi$, was reproducible for successive repeat experiments. And in the experiments, $\Phi$ is controlled in the range of $0.58-0.60$ by using tight compression.

Liquids and their properties used in the experiments are displayed in Table 1. Dimensionless parameters used in the analysis are Weber number $W e$, and the Reynolds number $R e: W e=\rho D u_{\mathrm{i}}^{2} / \sigma ; \operatorname{Re}=\rho u_{\mathrm{i}} D / \mu$; where $\rho, \mu, D$, and $\sigma$ are, respectively, liquid density, liquid dynamic viscosity, droplet impact diameter, and liquid surface tension factor; $\mathrm{g}$ is the gravitational acceleration and $u_{\mathrm{i}}=\sqrt{2 \mathrm{~g}\left(h_{\mathrm{r}}-D\right)}$ is the velocity at the time of impact. The droplet is released onto powder bed from different heights ranging from 3 to $600 \mathrm{~mm}$, resulting in impact velocity range from 0.2 to $3.2 \mathrm{~m} / \mathrm{s}$. Droplets can be considered as sphere upon impact. The equilibrium contact angle, $\theta$, is also measured.

The experimental setup (Fig. 1) includes a high-speed video camera (Qianyanlang 5KF20), which can capture the impact, spreading, and penetration in a single video sequence.

Table 1. Physical properties of liquids used.

\begin{tabular}{llllll}
\hline Liquid & $D(\mathrm{~mm})$ & $\rho\left(\mathrm{kg} \cdot \mathrm{m}^{-3}\right)$ & $\mu(\mathrm{mPa} \cdot \mathrm{s})$ & $\sigma\left(\mathrm{N} \cdot \mathrm{m}^{-1}\right)$ & $\theta\left(^{\circ}\right)$ \\
\hline Water & 2.10 & 997 & 1.003 & 0.072 & 86.60 \\
50\%ethanol & 1.78 & 900 & 1.10 & 0.0279 & 61.66 \\
Ethanol & 1.89 & 789 & 1.19 & 0.0232 & 66.9 \\
50\%glycerinum & 2.27 & 1120 & 4.21 & 0.070 & 57.62 \\
Glycerinum & 2.26 & 1260 & 1410 & 0.063 & 37.70 \\
20\% glycol & 2.42 & 1029 & 1.65 & 0.0605 & 66.00 \\
50\% glycol & 2.41 & 1073 & 3.94 & 0.056 & 63.87 \\
80\% glycol & 2.18 & 1110 & 10.05 & 0.0504 & 68.31 \\
Glycol & 2.17 & 1113 & 21.00 & 0.0484 & 58.23 \\
Acetone & 1.66 & 791 & 0.306 & 0.0237 & 72.77 \\
\hline
\end{tabular}

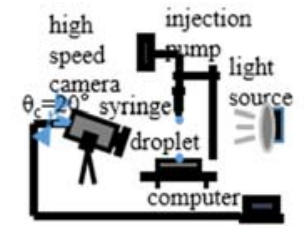

Fig. 1. Schematic of the experimental setup used.

\section{Results and discussion}

\subsection{Spreading time $t_{\mathrm{s}}$}

Fig. 2(a) depicts the spreading time $t_{\mathrm{s}}$ as a function of the impact velocity. The spreading time is typically a few milliseconds, which is of the same order of magnitude as the characteristic advective time ${ }^{[16]} \tau_{\mathrm{a}}=d_{0} /\left(2 u_{\mathrm{i}}\right)$ or the capillary-inertial time ${ }^{[17]} \tau_{\mathrm{i}}=\left(\rho d_{0}^{3} / 8 \sigma\right)^{0.5}$. Fig. 2(b) shows that $t_{\mathrm{s}}$ decreases sharply as the impact velocity increases for $W e^{*} \leq 40$ but slowly decreases for $W e^{*}>40$. Since $\tau_{\mathrm{a}}$ and $\tau_{\mathrm{i}}$ ignore influences such as viscosity and surface wettability, a modified capillary-inertia time $\tau_{\mathrm{i} \prime}=\left(\rho d_{\mathrm{max}}^{3} / 8 \sigma\right)^{0.5}$ is adopted to study the spreading ${ }^{[18]}$, where $d_{\max }$ is related to liquid viscosity and wettability. Fig. 2(c) shows a log$\log$ plot of normalized spreading time $t_{\mathrm{s}} / \tau_{\mathrm{i}}$, vs. $W e^{*}$. This shows that the function $t_{\mathrm{s}} / \tau_{\mathrm{i}}=$ $\zeta W e^{* \lambda}$ gives a good fit to the experimental data. The fitting coefficient $\zeta$ and the exponent $\lambda$ for different viscosities are shown in Fig. 2(d). The quantities $\zeta$ and $\lambda$ appear to be independent of liquid viscosity and substrate wettability. Consequently, a universal empirical expression, $t_{\mathrm{s}} /\left(\rho d_{\max }^{3} / 8 \sigma\right)^{0.5}=1.46 W e^{*-0.46}$, can accurately describe the spreading time in powder bed experiments. 

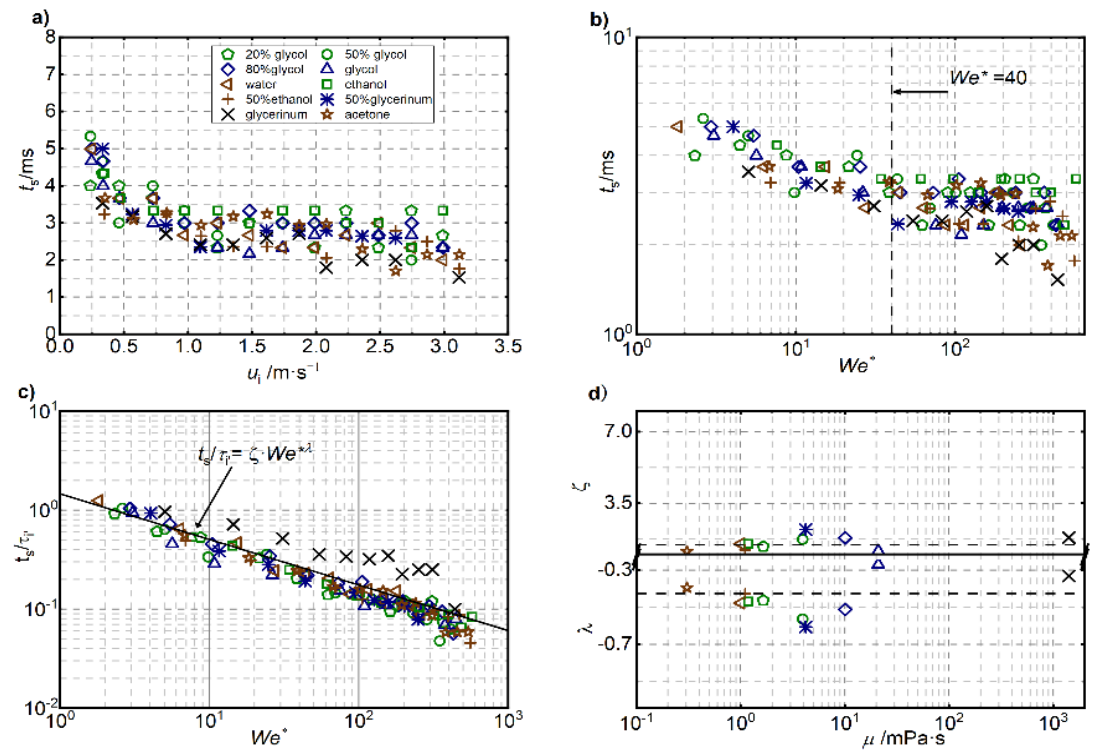

Fig. 2. (a) Spreading time $t_{\mathrm{s}} \mathrm{vs}$. impact speed $u_{\mathrm{i}}$. (b) Spreading time $t_{\mathrm{s}}$ vs. effective Weber number $W e^{*}$. (c) Normalized spreading time $t_{\mathrm{s}} / \tau_{\mathrm{i}}$, vs. $W e^{*}$. (d) The coefficient $\zeta$ and exponent $\lambda$ of the graph in part (c) vs. liquid viscosity.
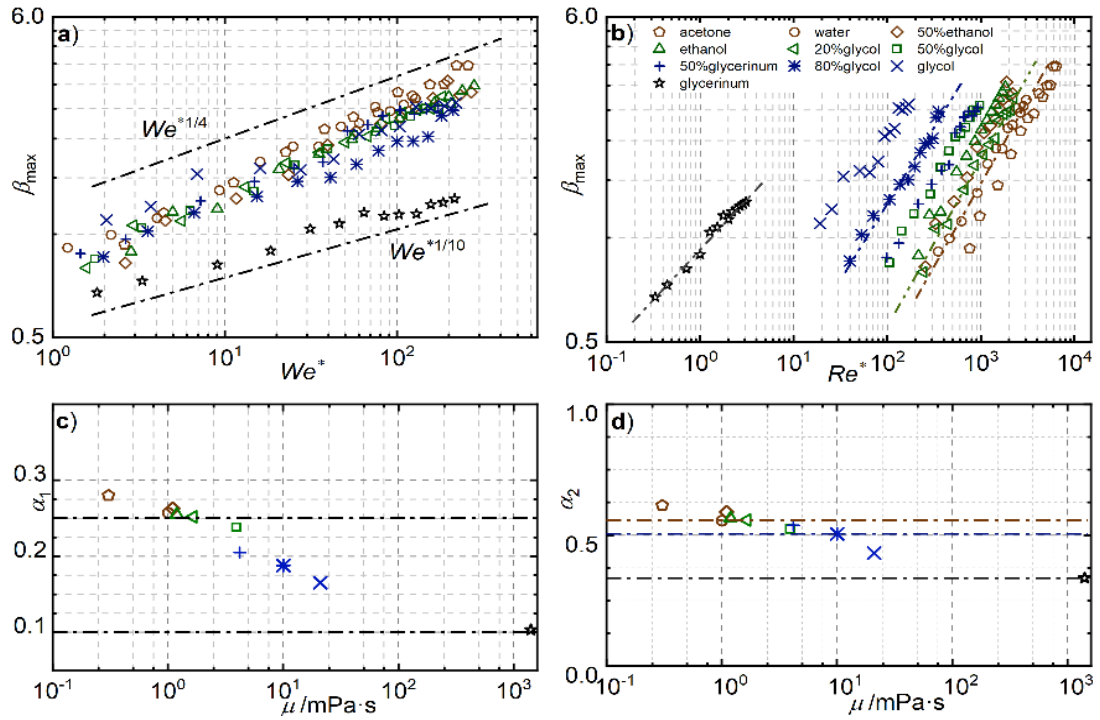

Fig. 3. The maximum spreading factor $\beta_{\max }$ vs (a) $W e^{*}$ and (b) $R e^{*}$ for all liquids. The maximum spreading factors were fitted in (a) with $\beta_{\max }=A_{1} W e^{* \alpha 1}$ and in (b) with $\beta_{\max }=A_{2} R e^{* \alpha 2} \cdot \alpha_{1}$ and $\alpha_{2}$ as functions of liquid viscosity are plotted in (c) and (d).

\subsection{Maximum spreading factor $\boldsymbol{\beta}_{\max }$}

An effective Weber number $W e^{*}=\left[D /\left(D+2 Z_{\mathrm{m}}\right)\right] W e$, where $Z_{\mathrm{m}}$ is the maximum vertical deformation measured ${ }^{[11]}$, is used to replace $W e$. It has been shown that $W e^{*}$ collapses the maximum spreading data for various packing densities ${ }^{[19]}$, indicating that $W e^{*}$ can also characterize the effect between liquid droplets with various viscosity and the powder bed 
with the same packing fraction. Fig. 3 gives the correlation of $\beta_{\max } \mathrm{vs} W e^{*}\left(W e^{*}=[D /(D+\right.$ $\left.\left.\left.2 Z_{\mathrm{m}}\right)\right] W e=W e^{I}\right)$ and the correlation of $\beta_{\max }$ vs $R e^{*}\left(R e^{*}=\left[D /\left(D+2 Z_{\mathrm{m}}\right)\right] R e \neq R e^{I}\right.$, because the value of $\frac{R e}{R e^{I}}=\frac{\rho v_{p}}{\mu}=\frac{2(1-\phi)^{4} d_{g}^{2}\left[\frac{2 \sqrt{6}\left(D+2 Z_{m}\right) \sigma^{0.5} \rho^{1.5} u_{i} D^{-1.5}}{\sqrt{\pi}}+\frac{4 \rho \sigma \cos \theta}{d}\right]}{\left[\mu^{2}\left(180 \phi^{2}\right)\right]}$ depends heavily on viscosity, $R e^{I}$ is not applicable to the analysis of viscosity effect in this paper, Fig. 4 also proves this point) respectively for various viscosity liquids. As shown in Fig. 3 (a) and (c), a scaling law behavior, $\beta_{\max } \sim W e^{* \alpha 1}$, is observed at $W e^{*}>1$. It comes as no surprise to see that $\beta_{\max }$ increases with $W e^{*}$. The data set exhibits three other distinguished features: (1) The maximum spreading factor is suppressed with increasing viscosities (different color symbols in Fig. 3 (a)). (2) In the doubly logarithmic scale, there are two asymptotes in the extension of data: the upper is $W e^{* 1 / 4}$ and the lower is $W e^{* 1 / 10}$. (3) The value of $\alpha_{1}$ decreases with increasing viscosities (Fig. 3(c)). On the other hand, it seems that the spreading is dominated by viscous force within the liquid for all experimental data. In Fig. 3(b), $\beta_{\max }$ grows with $R e^{*}$ following the power law, $\beta_{\max } \sim R e^{* \alpha 2}$. The exponent $\alpha_{2}$ is $\sim 0.5$ for lowly viscous liquids with $\mu \leq 10 \mathrm{mPa} \cdot \mathrm{s}$, and it decreases to $\sim 0.33$ for liquids with $\mu=1430 \mathrm{mPa} \cdot \mathrm{s}$.

Because of the quantitative relation $W e^{1 / 10} \sim D^{-1 / 4} R e^{1 / 5}$, a law of the type $\beta_{\max } \sim W e^{* 1 / 10}$ leads to $\beta_{\max } \sim R e^{1 / 5}$. According to the analysis of the transition to a viscous regime, we thus define the effective number $P^{*} \equiv W e^{*} / R e^{* 4 / 5}$ for deformable substrate $\left(P \equiv W e / R e^{4 / 5}\right.$ for non-deformable substrate $\left.{ }^{[20]}\right)$. The transition between the capillary regime and viscous regime is plotted in Fig. 4(a), where the dimensionless viscous extension $\beta_{\text {max }} / R e^{* 1 / 5}$ is plotted as a function of $P^{*}$. While for $P^{\dagger}=W e^{\dagger} / R e^{\dagger 4 / 5}$ in Fig. 4(b), the result is surprisingly that the transition is opposite to Fig. 4(a) - the viscous droplet spreading changes from viscous regime to the capillary regime as $P^{\dagger}$ increases. Consequently, $R e^{\dagger}-$ incorporates the bulk wettability of a granular substrate - doesn't fit to interpret the influence of liquid viscosity on spreading. The use of $W e^{*}$ and $R e^{*}$ reveals the hidden similarities between a droplet impact on deformable substrate and that on a non-deformable substrate for maximum droplet spreading. Same as the non-deformable substrate, the influence of internal inertial flow at maximum spreading phase also exists, and causes the maximum spreading smaller than the theoretical value.
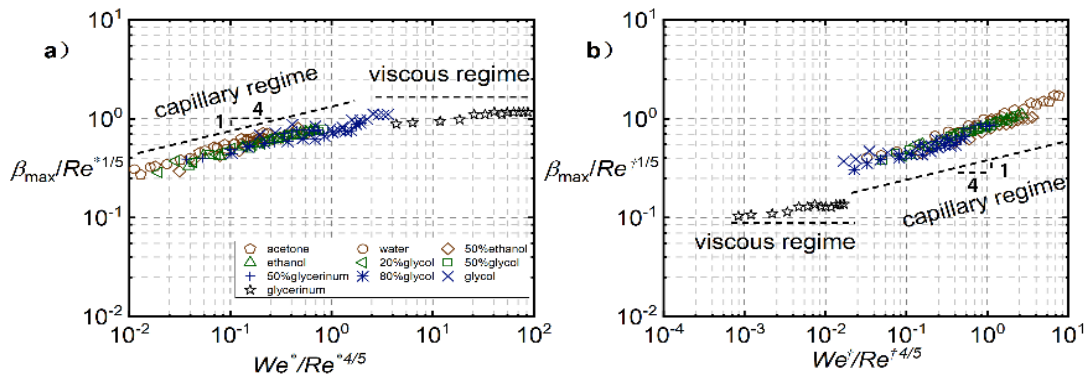

Fig. 4. (a) The maximum spreading factor $\beta_{\max }$ of Fig. 3 in a doubly logarithmic plot. (b) is based on (a), but $W e^{*}$ and $R e^{*}$ are replaced by $W e^{\dagger}$ and $R e^{\dagger}$.

\section{Conclusions}

We have performed an experimental study of various liquid droplets impacting alumina powder beds. Weber numbers $(W e)$ and Reynolds numbers $(R e)$ were obtained by adjusted the droplet fall height. Ten liquids were used that provided various viscosities and various surface tensions.

Then we adopted spreading time $t_{\mathrm{s}}$ and maximum spreading factor $\beta_{\max }$ to quantitatively analyze the spreading behavior of the droplets after impacting the powder bed. The modified 
capillary-inertial time $\tau_{\mathrm{i}}=\left(\rho d_{\max }^{3} / 8 \sigma\right)^{0.5}$ considering the influence of substrate wettability and liquid viscosity, and the effective Weber number $W e^{*}=\left[D /\left(D+2 Z_{\mathrm{m}}\right)\right] W e$ considering the influence of the deformable substrate, are innovatively put forward to achieve a universal scaling law: $t_{\mathrm{s}} /\left(\rho d_{\max }^{3} / 8 \sigma\right)^{0.5}=1.46 W e^{*-0.46}$. Besides, the effective weber number $W e^{*}$, replacing traditional weber number, is adopted to attain the scaling law $\beta_{\max } \sim W e^{* \alpha 1}$, and $\alpha_{1}$ decreases with the increasing viscosity. The same thing happens for the scaling law $\beta_{\max } \sim R e^{* \alpha 2}$. The capillary regime considered implies a small $P^{*}\left(P^{*}=\right.$ $\left.W e^{*} / R e^{* 4 / 5}<1\right)$, the viscous regime refers to large $P^{*}\left(P^{*}=W e^{*} / R e^{* 4 / 5}>10\right)$. Thus, the use of $W e^{*}$ and $R e^{*}$ reveals the hidden similarities between a droplet impact on deformable substrate and that on a non-deformable substrate for maximum droplet spreading.

This study is supported by Grants from National Natural Science Foundation of China (No. 51876071).

\section{References}

1. Hapgood, K. P. \& Khanmohammadi, B. Powder Technol. 189, 253-262 (2009).

2. Knight, P. C. Powder Technol. 119, 14-25 (2001).

3. Kayrak-Talay, H. N. E. and D. J. D. L. IFAC Proc. Vol. (2012) doi:10.1002/aic.

4. Moreira, A. L. N., Moita, A. S. \& Panão, M. R. Prog. Energy Combust. Sci. 36, 554-580 (2010).

5. Sachs, E., Cima, M., Williams, P., Brancazio, D. \& Cornie, J. J. Manuf. Sci. Eng. Trans. ASME 114, 481-488 (1992).

6. van Dam, D. B. \& Le Clerc, C. Phys. Fluids 16, 3403-3414 (2004).

7. Van Der Bos, A. et al. Phys. Rev. Appl. 1, 1-9 (2014).

8. Rioboo, R., Marengo, M. \& Tropea, C. Exp. Fluids 33, 112-124 (2002).

9. van der Meer, D. Annu. Rev. Fluid Mech. 49, 463-484 (2017).

10. Delon, G., Terwagne, D., Dorbolo, S., Vandewalle, N. \& Caps, H. Phys. Rev. E - Stat. Nonlinear, Soft Matter Phys. 84, 3-7 (2011).

11. De Jong, R., Zhao, S. C., Garcia-Gonzalez, D., Verduijn, G. \& Van Der Meer, D. Soft Matter 17, 120-125 (2021).

12. Hardt, S. \& McHale, G. Annu. Rev. Fluid Mech. 54, 83-104 (2022).

13. Li, S. et al. Science Advances vol. 7 (2021).

14. Graeber, G. et al. Nat. Commun. 12, 1-7 (2021).

15. Yarin, A. L. Annu. Rev. Fluid Mech. 38, 159-192 (2006).

16. Supakar, T., Moradiafrapoli, M., Christopher, G. F. \& Marston, J. O. J. Colloid Interface Sci. 468, 10-20 (2016).

17. Wildeman, S., Visser, C. W., Sun, C. \& Lohse, D. J. Fluid Mech. 805, (2016).

18. Lin, S. et al. J. Colloid Interface Sci. 516, 86-97 (2018).

19. Zhao, S. C., De Jong, R. \& Van Der Meer, D. Soft Matter 11, 6562-6568 (2015).

20. Clarke, A., Blake, T. D., Carruthers, K. \& Woodward, A. Langmuir 18, 2980-2984 (2002). 